\title{
Wiener Filter based Medical Image De-noising
}

\author{
Dr. Sana'a khudayer Jadwa \\ Assist. Prof. \\ Computers Unit \\ College of Medicine \\ Baghdad- Iraq
}

\begin{abstract}
Medical images such as , CT (Computed Tomography) scan imaging and MRI (magnetic resonance imaging) are considered as a collection of information that is used for visual diagnostics .Most of the medical images are affected by different types of noises during acquisition, storage and transmission, so the information associated with an image tends to loss or damage that can affect the quality of disease diagnosis or treatment. Image de-noising is the process to remove the noise from the image naturally corrupted by the noise. In this paper an effective noise reduction approach based on using Wiener filter is proposed to enhance the image qualities of various medical imaging modalities
\end{abstract}

Keywords: Wiener filter; Image de-noising; Image processing; Medical Imaging; Noise.

\section{INTRODUCTION}

In medical science, the image processing techniques is playing an important role; these techniques are the reliable diagnosis tools in medical sciences. It is used for detecting cracks in bones and soft tissues like liver, kidney, spleen, uterus, heart, brain etc. These methods of diagnosis are widely acceptable because they are inexpensive, harmless to human body, portable and noninvasive. Another advantage of these techniques is that these are very fast [1].The advent of digital imaging technologies such as MRI has revolutionized modern medicine. Today, many patients no longer need to go through invasive and often dangerous procedures to diagnose a wide variety of illnesses. With the wide spread use of digital imaging in medicine today, the quality of digital medical images becomes an important issue. To achieve the best possible diagnoses it is important that medical images be sharp, clear, and free of noise and artifacts. While the technologies for acquiring digital medical images continue to improve, resulting in images of higher and higher resolution and quality, noise remains an issue for many medical images. Removing noise in these digital images remains one of the major challenges in the study of medical imaging[2]. Noise is introduced in the medical images due to various reasons. In medical imaging, noise degrades the quality of images. This degradation includes suppression of edges, blurring boundaries etc. [3]. Image de-noising has become an essential exercise in medical imaging especially the Magnetic Resonance Imaging (MRI). In recent years, technological development has significantly improved in analyzing medical images. Medical image enhancement has attracted much attention during the diagnosis process. Enhanced medical images are desired by a surgeon to help diagnosis and interpretation because medical image qualities are often deteriorated by artifacts. Nowadays Medical imaging is the best techniques for monitoring the person's diagnosis process. Most of the diseases are diagnosed by doctors using medical imaging methods. One problem that physician encounter because of the low quality of medical image, this low quality causes difficulty during the diagnosis. So it is necessary to improve the quality of the medical image [4]. In order to improve the quality of images, there are various filtering techniques used in image processing. There are various filters which can remove the noise from images and preserve image details [5]. This paper produce Wiener filter for medical images de-noising. The organization of the rest of this paper is as follows. Section 2 highlights the related works. Section 3 introduces image denoising. Section 4 describes the Wiener Filter. The proposed method introduced in section 5. Section 6 presents the experimental results and section 7 concludes the paper.

\section{RELATED WORKS}

The image de-noising naturally corrupted by noise is a classical problem in the field of signal or image processing.[6] proposed de-noising method of medical images through thresholding and optimization using a randomized and stochastic technique of Particle Swarm Optimization(PSO) algorithm. PSO are population based optimization algorithm, which is initialized with a group of random particles and then searches for optima by updating generations.[7] In this paper, an algorithm for image denoising was designed to develop K-SVD by using Regularized Orthogonal Matching Pursuit (ROMP) over log Gabor wavelet adaptive dictionary.[8] In this paper Discrete Wavelet transform is used for image denoising as it allows multiresolution decomposition. The wavelet coefficients are threshold using hard and soft thresholding techniques. This paper proposed a novel method of medical images de-noising through thresholding and optimization using Genetic Algorithm (GA). [9] In this paper, a novel global noise reduction approach based on the sparse representation and nonlocal means algorithm is proposed to enhance the image qualities of various medical 
imaging modalities including ultrasound images and magnetic resonance images. By using an over complete dictionary, a medical image is decomposed into a sparsest coefficients matrix populated primarily with zeros. A nonlocal means algorithm is developed to deal with these sparse coefficients to exploit the repetitive characters of structures in the whole image, realizing a "truly" global de-noising.[10] A new signal-preserving technique for noise suppression in event-related magnetic resonance imaging (MRI) data is proposed based on spectral subtraction. Simple form, the new method does not change the statistical characteristics of the signal or cause correlated noise this suggests the new technique as a useful preprocessing step for MRI data analysis.

\section{IMAGE DE-NOISING}

Image de-noising is an important image processing task both as a process itself, and as a component in other processes. many ways to de-noise an image or a set of data exists. Image de-noising still remains a challenge for researchers because noise removal introduces artifacts and causes blurring of the images [6].Image de-noising is a kind of processing of image which belongs to image restoration, and the ultimate goal of restoration techniques is to improve an image in some predefined sense. So de-noising is the key step of image processing and recognition [11].An image is often corrupted by noise in its acquition and transmission. Image de-noising is used to remove the additive noise while retaining as much as possible the important signal features. [12].There is many schemes for removing noise from images. The good de-noising scheme must able to retrieve as much of image details even though the image is highly affected by noise[13].In case of image denoising methods, the characteristics of the degrading system and the noises are assumed to be known beforehand. The image $s(x, y)$ is blurred by a linear operation and noise $n(x, y)$ is added to form the degraded image $w(x, y)$. This is convolved with the restoration procedure $g(x, y)$ to produce the restored image $z(x, y)$. The "Linear operation" shown in Figure 1, is the addition or multiplication of the noise $n(x, y)$ to the $\operatorname{signal}^{s(x, y)}$. Once the corrupted image $w(x, y)$ is obtained, it is subjected to the de-noising technique to get the de-noised image $z(x, y)$ [14].

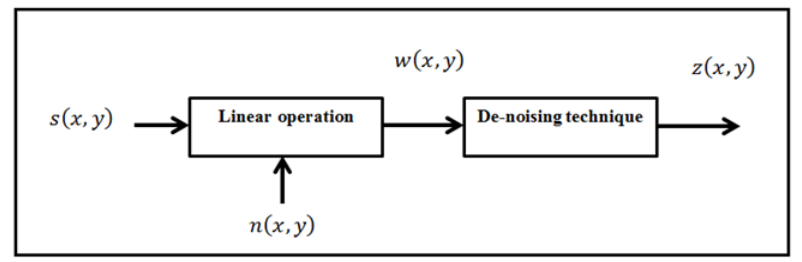

Figure 1. De-noising Concept.

\subsection{Noise}

Noise is basically a disturbance that distorts the information present in the image. It is usually an unwanted signal that can create a variation in image intensity levels of pixels which cause degradation of image quality. The noise is introduced in image automatically due to following reasons:

Digital Image Acquisition Process: In acquisition process, the optical image is converted into a series of electronic signals. During this process, unwanted signals may be added into original series of electronic signals there may be certain other unavoidable situations such as mechanical problem, out of focus blur, motion, in appropriate illumination.

Image Transmission Process: The most of time noise is added during transmission process such as scanning of image using scanner, converting one image format into another format, wireless network transmission of image etc. During transmission process, noisy channel and error due to measurement process may introduce unwanted signals into data stream which results in noised image.

Sensitivity of Image Sensors: The image sensors are sensitive to motion and cause noising during capturing process due to malfunctioning of pixel elements in the camera sensors, faulty memory locations, or timing errors in the digitization process [15].

\subsection{Types of Noise}

There are many types of noises occurs in medical images. Mostly occurred noise is: Gaussian noise, Speckle noise, Salt and pepper noise.

\subsubsection{Gaussian Noise}

Gaussian noise has a Gaussian distribution, with a bell shaped distribution and probability function given by:

$f(g)=\frac{1}{\sqrt{2 \pi \sigma^{2}}} e^{-(g-m)^{2}} / 2 \sigma^{2}$

where $\mathrm{g}$ represents the gray level, $\mathrm{m}$ is the mean or average of the function, and $\sigma$ is the standard deviation of the noise[16].

\subsubsection{Salt and Pepper Noise}

Salt and pepper noise is an impulse type of noise, and caused due to errors in data transmission. It has only two possible values, low and high.. The corrupted pixels are set alternatively to the minimum or to the maximum value, giving the image a "salt and pepper" like appearance. Unaffected pixels remain unchanged. For an 8-bit image, the typical value for pepper noise is 0 and for salt noise is $255[16]$.

\subsubsection{Speckle Noise}

For Speckle noise is a repetitive type of noise and occurs in imaging systems such as laser and SAR (Synthetic Aperture Radar) . The source of this noise is attributed to random interference between the coherent returns. The 
Mathematical expression for this noise is given by:

$f(g)=\frac{g^{\alpha-1}}{(\alpha-1) ! a^{\alpha}} e^{-\frac{g}{a}}$

Where $\boldsymbol{a}^{\alpha}$ is variance and $g$ is the gray level.

\section{WIENER FILTER}

Wiener filter is proposed by Norbert Wiener in 1940 and published in 1949. It is used to reduce noise in signal. When the image is blurred by a known low pass filter, it is possible to recover the image by inverse filtering. But inverse filtering is very sensitive to additive noise. The Wiener filtering executes an optimal trade-off between inverse filtering and noise smoothing. It removes the additive noise and inverts the blurring simultaneously. The Wiener filter minimizes the mean square error between the estimated process and the desired process. It minimizes the overall mean square error in the process of inverse filtering and noise smoothing. The Wiener filtering is a linear estimation of the original image [17].The important use of Wiener filter is to reduce the amount of noise present in an image by comparison with an estimation of the desired noiseless signal. It is based on a statistical approach. Wiener filters are characterized by three important factors.

1-Assumption: stationary linear stochastic processes of image and noise with known spectral characteristics or known autocorrelation and cross correlation.

2-Requirement: the filter must be physically realizable/ causal.

3-Performance criterion minimum mean-square error (MMSE). This filter is frequently used in the process of deconvolution[18].

The Wiener filter is commonly utilized because of its simplicity and its speed. It is deemed simple because it uses a system of linear equations to calculate a set of optimal filter weights that reduce the noise level of a received signal. It estimates cross-correlation and covariance matrices of noisy signals to calculate these weights and provide an accurate Estimate of the undistorted deterministic signal under Gaussian noise. The noise statistics are estimated and then used to determine a set of optimal filter weights. By then processing a new input signal, containing similar noise characteristics with the optimal filter weights, the signal deterministic component is estimated. This method is optimal when the noise distribution is Gaussian. Furthermore, its execution only requires a few computational steps that are very fast to process[19].

Wiener Filter in the Fourier Domain:

$G(u, v)=\frac{H^{*}(u, v) P_{s}(u, v)}{|H(u, v)|^{2} P_{s}(u, v)+P_{n}(u, v)}$

Dividing through by $P_{s}$ makes its behavior easier to explain :

$$
G(u, v)=\frac{H^{*}(u, v)}{|H(u, v)|^{2}+\frac{P_{n}(u, v)}{P_{s}(u, v)}}
$$

Where :

$H(u, v)=$ Degradation function

$H^{*}(u, v)=$ Complex conjugate of degradation function

$P_{n}(u, v)=$ Power Spectral Density of Noise

$P_{s}(u, v)=$ Power Spectral Density of un-degraded image

The term $\frac{\boldsymbol{P}_{\boldsymbol{n}}}{\boldsymbol{P}_{\boldsymbol{S}}} \quad$ can be interpreted as the reciprocal of the signal -to-noise ratio [20].

The Wiener filter is used to remove the noise from a corrupted found to image based on statistics estimated from a local neighborhood of each pixel . This filter depends on noise power (i.e. noise variance in a corrupted image). When the variance is large, the filter performs little smoothing and when the variance is small, the filter performs more smoothing[21].

\section{PROPOSED METHOD}

In this paper a de-noising approach for medical images based on applying a Wiener filter is proposed. At first the color image is read from a database that contain a collection of medical images, then these images are converted to grayscale images, later the Wiener filter is applied in order to remove the noise from the medical image. The Wiener filter de-noising process diagram is illustrated in Figure 2, as shown below:

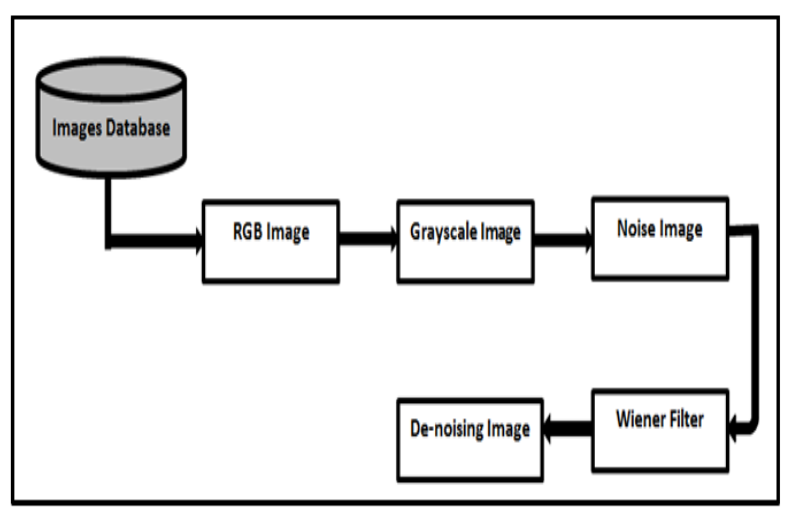

Figure 2 :Process of image De-noising based on Wiener filter 


\subsection{Images Database}

A database for the proposed method containing six medical images (lung, Stomach, and liver) images, are collect from the web with different sizes. As shown in Figure 3:

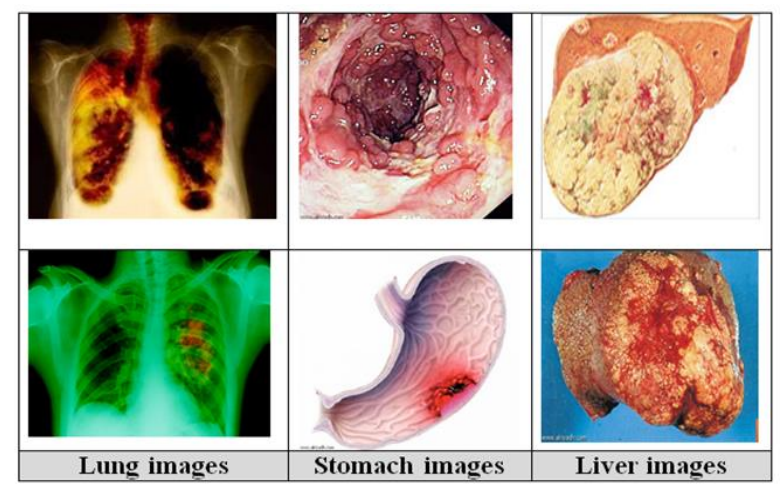

Figure 3 : Images Database

\subsection{RGB Image Conversion}

The input image is coloured medical image that is converted to grayscale image by converts RGB values to grayscale values by forming a weighted sum of the R, G, and $B$ components using Eq.(5):

$$
y=0.2989 R+0.5870 G+0.1140 B
$$

\subsection{Noise Image}

The noisy image is produced by adding the Gaussian noise to the grayscale image, Gaussian noise is statistical noise having a probability density function (PDF) equal to that of the normal distribution, which is also known as the Gaussian distribution. In other words, the values that the noise can take on are Gaussian-distributed.

\subsection{Wiener Filter}

By using the wiener2 function to remove the noise from noisy image. The Wiener filter tailors itself to the local image variance. Where the variance is large, wiener 2 performs little smoothing. Where the variance is small, wiener 2 performs more smoothing.

\section{EXPERIMENTAL RESULTS}

The experiments of the proposed Wiener filter method is realized by Matlab software and are implemented on Intel Core i7-2330M CPU, $2.20 \mathrm{GHz}$ with $2 \mathrm{~GB}$ RAM under Windows 8 platform. The coloured medical image first is reading from the database then the RGB image is converted to grayscale as shown in Figure 4:

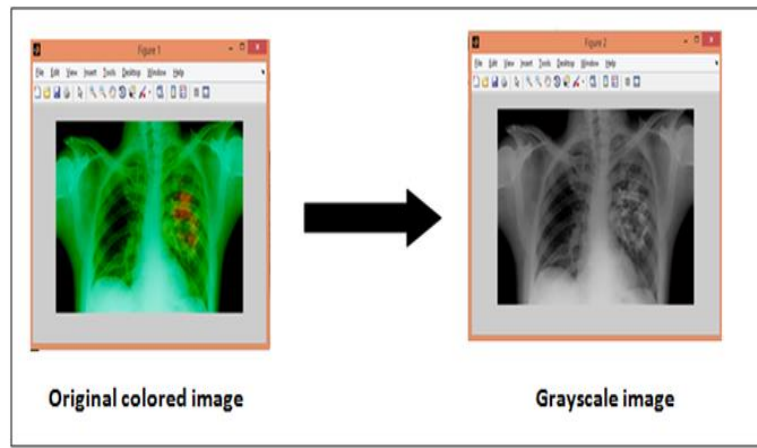

Figure 4: Colored Image Conversion.

Then, adding Gaussian noise with "0" as mean and " 0.025 " as standard variance to grayscale image, which is shown in Figure 5:

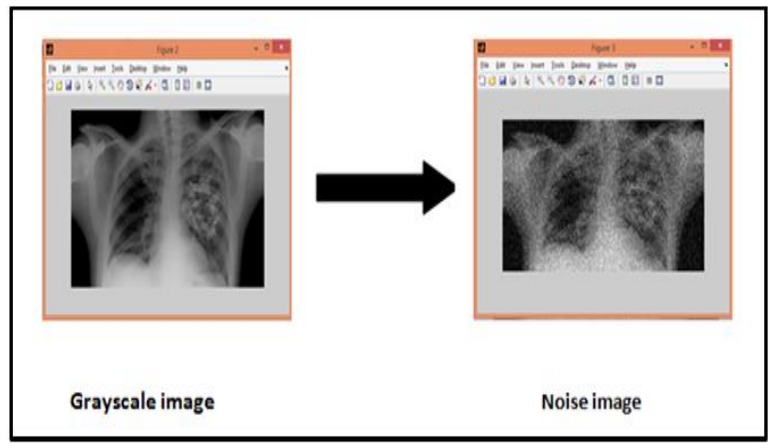

Figure 5: Noisy Image $(\sigma=0.025)$

Finally the de-noising image is produced by using the wiener2 function, as shown in Figure 6:

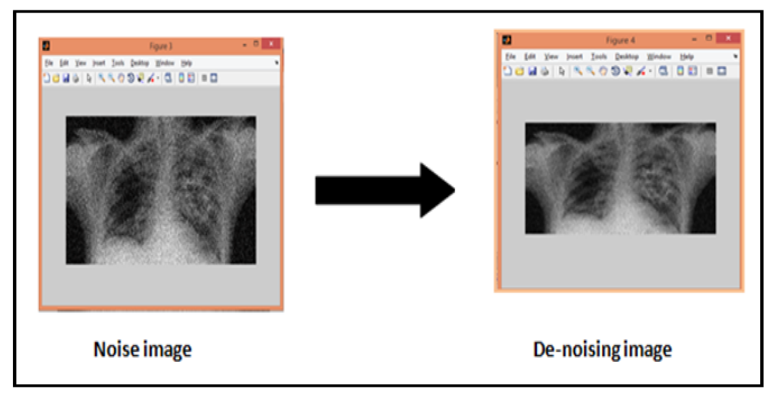

Figure 6: The De-noising Image

After processing, the de-noised experimental results of noised image can be obtained by simulation, which are shown in Figure 7, respectively: 


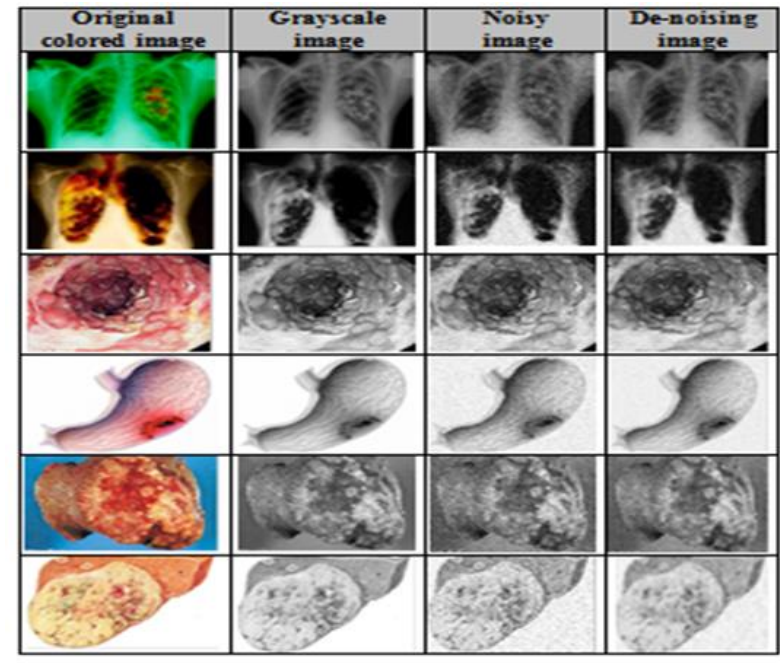

Figure 7: Results for the De-noising of Medical Images

\section{CONCLUSION}

Medical images are often affected by noise because of both image acquisition from the medical modalities and image transmission from modalities to workspace in the main computer system. This noisy usually affects the visual quality of the original images so image de-noising always has been issued in the medical image processing to make the diagnosing more efficient. In this work we have introduced a relatively simple and efficient method for removal of Gaussian noises from Medical Images using the Wiener filter. Wiener filter is an excellent filter when it comes to noise reduction or de-blurring of images. It considers both the degradation function and noise as part of analysis of an image. The experimental results reveals Wiener filter robustness and detail preservation. The Wiener filter is commonly utilized because of its simplicity and its speed. It is deemed simple because it uses a system of linear equations to calculate a set of optimal filter weights that reduce the noise level of a received signal. The resulting figure show the efficiency, simplicity and robustness of medical image de-noising. Wiener filtering has the advantages of small calculation and good noise effect, so it has been used widely. Many efficient de-noising algorithms are based on the principle of Wiener filtering, whose purpose is to restore the original image and reach the minimum mean error with the original image.

\section{REFERENCES}

[1] Jagdeep Kaur †* and Ruchika Manchanda, (Feb 2015)," Optimizing Wavelet based Medical Image De-Noising", International Journal of Current Engineering and Technology, Vol.5, No.1.

[2 ]Prakash B. L. , Vudimudi R. B.,2014," Performing Various Image Denoising Techniques for Medical Images" ,International Journal of Advance Research in Computer Science and Management Studies, Volume 2, Issue 1.
[3] Senthilraja S., Dr. Suresh P., Dr. Suganthi M., March2014, " Noise Reduction in Computed Tomography Image Using WB - Filter", International Journal of Scientific \& Engineering Research, Volume 5, Issue 3 .

[4] Deepa B. and Sumithra M. G., 2015," MRI Medical Image Denoising by Combined Spectral Subtraction and Wavelet based Methods", ARPN Journal of Engineering and Applied Sciences, VOL. 10, NO. 4.

[5] Malothu Nagu, Shanker N.V.,2014 " Image De-Noising By Using Median Filter and Weiner Filter", International Journal of Innovative Research in Computer and Communication Engineering, Vol. 2, Issue 9.

[6] Ms. Dansena1P., Mr. Dewangan O., 2015, " Adaptive Thresholding for Wavelet Denoising on Medical Images through PSO Algorithm", International Journal of Advanced Research in Computer Engineering \& Technology (IJARCET) Volume 4 Issue 5.

[7] Farouk R.M., Elsayed M. and Aly M., 2016," Medical Image De-noising based on Log-Gabor Wavelet Dictionary and K-SVD Algorithm", International Journal of Computer Applications (0975 - 8887 Volume 141 - No.1.

[8] Singh S., Wadhwani S. , 2015," Genetic Algorithm Based Medical Image Denoising Through Sub Band Adaptive thresholding", International Journal of Science, Engineering and Technology Research (IJSETR), Volume 4, Issue 5.

[9] Guo Yi, Hanchao C. , Yuanyuan W.,2014," A Global Approach for Medical Image Denoising via Sparse Representation", International Journal of Bioscience, Biochemistry and Bioinformatics.

[10] Priyadharsini.B, Vanitas, 2014,"Denoising MRI Images Using A Non-Linear Digital Filter", International Journal of Advanced Research in Electronics and Communication Engineering (IJARECE)Volume 3, Issue 4.

[11] Mr. Kethwas A., Dr. Jharia B. , 2014,"Comparison Study on Image Denoising Through Wiener Filter", International Journal of Engineering Research \& Technology (IJERT )Vol. 3 , Issue 8 .

[12] Mondal T., Dr. Maitra M. , 2014," Denoising and Compression of Medical Image in Wavelet 2D", International Journal on Recent and Innovation Trends in Computing and Communication Volume 2, Issue 2.

[13] Govindaraj V., Sengottaiyan G.,2013," Survey of Image Denoising using Different Filters", International Journal of Science, Engineering and Technology Research (IJSETR )Volume 2, Issue 2. 
[14] Chandrika S., Prof. Deepak K. ,2014, " Noises and Image Denoising Techniques: A Brief Survey", International Journal of Emerging Technology and Advanced Engineering Volume 4, Issue 3.

[15] Pathak M. , 2014, " COMPARATIVE ANALYSIS OF IMAGE DENOISING TECHNIQUES", Monika Pathak et al. ,International Journal of Computer Science \& Engineering Technology (IJCSET), Vol. 5 No. 02.

[16] Tamilselvan K.S., Murugesan G., and Vinothsaravanan M, 2013, " A Histogram based Hybrid Approach for Medical Image Denoising using Wavelet and Curvelet Transforms", International Journal of Computer Applications (0975 - 8887) Volume 74- No. 21.

[17] Jaspreet k., Rajneet k. , 2013," Biomedical Images denoising using Symlet Wavelet with Wiener filter ", International Journal of Engineering Research and Applications,Vol.3,Issue 3.

[18] Umapathi V.J., Narayanan V.,2014," MEDICAL IMAGE DENOISING BASED ON GAUSSIAN FILTER AND DWT SWT BASED ENHANCEMENT TECHNIQUE", International Journal of Soft Computing and Artificial Intelligence,Volume-2, Issue-2.

[19] Anilet B., Chiranjeeb H. and Punith C.,2014," Image Denoising Method Using curvelet Transform and Wiener Filter", International Journal of Advanced Research in Electrical Electronics and Instrumentation Engineering, Vol. 3, Issue 1.

[20]Suresh K., Papendra K., Manoj G., and Ashok K. N.,2010, " Performance Comparison of Median and Wiener Filter in Image De-noising", International Journal of Computer Applications, Volume 12- No.4.

[21] Sarungbam B. and Yambem J.,2015, "Denoising of Image by Wiener Filtering in Wavelet Domain", Advanced Research in Electrical and Electronic Engineering, Volume 2, Issue 11. 